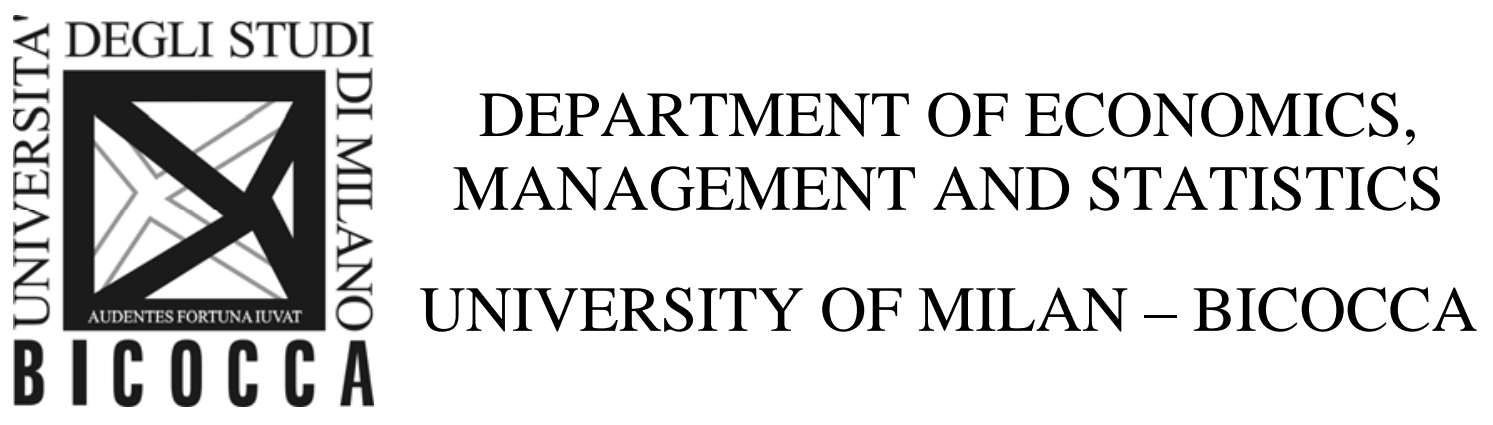

DEMS WORKING PAPER SERIES

\title{
An adaptive decisional mechanism leading to chaos
}

Ahmad Naimzada, Marina Pireddu

No. 252 - July 2013

Dipartimento di Economia, Metodi Quantitativi e Strategie di Impresa Università degli Studi di Milano - Bicocca http://dems.unimib.it/ 


\title{
An adaptive decisional mechanism leading to chaos
}

\author{
Ahmad Naimzada ${ }^{\text {a* }}$, Marina Pireddu ${ }^{\text {b } \dagger}$ \\ ${ }^{a}$ Dept. of Economics, Quantitative Methods and Management, University of Milano-Bicocca, Milan \\ ${ }^{\mathrm{b}}$ Dept. of Mathematics and Applications, University of Milano-Bicocca, Milan
}

\begin{abstract}
In this paper we propose a framework in order to analyze the dynamical process of decision and opinion formation of two economic homogeneous and boundedly rational agents that interact and learn from each other over time. The decisional process described in our model is an adaptive adjustment mechanism in which two agents take into account the difference between their own opinion and the opinion of the other agent. The smaller that difference, the larger the weight given to the comparison of the opinions. We also assume that if the distance between the two opinions is larger than a given threshold, then there is no interaction and the agents do not change their opinion anymore. Introducing an auxiliary variable describing the distance between the opinions, we obtain a one-dimensional map for which we investigate, mainly via analytical tools, the stability of the steady states, their bifurcations, as well as the existence of chaotic dynamics and multistability phenomena, i.e., the presence of coexisting attractors.
\end{abstract}

Keywords: adaptive decisional mechanism; bifurcations; multistability; complex dynamics.

AMS classification: 37N40, 39A28, 39A60, 91B55

JEL classification: D03, D79, D83

\footnotetext{
*Mailing address: University of Milano-Bicocca, U7 Building, Via Bicocca degli Arcimboldi 8, 20126 Milano, Italy. Tel.: +390264485813; fax: +390264483085. E-mail address: ahmad.naimzada@unimib.it

${ }^{\dagger}$ Corresponding author at: University of Milano-Bicocca, U5 Building, Via Cozzi 53, 20125 Milano, Italy. Tel.: +39 0264485729; fax: +390264485705. E-mail address: marina.pireddu@unimib.it
} 


\section{Introduction}

Traditionally economic theory has been based on models with fully rational representative agents with preferences that are constant over time and clearly defined intertemporal budget constraints. Although the theoretical advances obtained by such an approach are widely recognized, some authors have proposed the possibility of extending the theory in order to incorporate a more dynamical perspective, which is not well developed in the traditional economic formalization $[14,22]$. Moreover, some authors believe it is desirable to revise some of the assumptions of perfect rationality, by allowing more bounded types of rational behavior and learning mechanisms [19]. The introduction of this kind of aspects produces in fact models describing more realistically some features of the decision making process and opinion dynamic formation. For instance, in the case of duopoly modeling, instead of full rationality implying the players to choose directly the Nash equilibrium, it is often assumed that agents are boundedly rational and adopt decisional rules such as best response adjustment and gradient mechanisms [5, 12, 23].

In this work our contribution concerns an approach that is based on the idea that economic and social decisions are the result of social interactions of homogeneous and boundedly rational agents that interact and learn from each other over time. In our model the dynamical process is not explained by optimal intertemporal trajectories that are defined by constant preferences and well-defined budget constraints, but rather by the behavior of homogeneous agents that interact with each other, adapting their decisions in accordance with the other players' choices. Our model conforms with some approaches analyzing the motivations of decision making within disciplines such as sociology, psychology and marketing [2]. In the present paper we assume that there exist emulation and learning from interaction with other individuals. We do not consider what is called intrinsic utility of the decision process, that is, utility derived from the economic and social activities. We take into account only external influences derived from the observation of the others' behavior. We adopt such an approach in order to highlight the role of social interaction as a source of continuous update of the decisional process.

More precisely, in the present paper we propose a framework with two interacting agents, in which each agent weights the decision or the opinion of the other agent to a certain extent in forming his own new decision or opinion. This process is modeled by an adaptive adjustment mechanism. In particular, similarly to what assumed in [15], the weight with which an agent takes into account the difference between his own opinion and the opinion of the other agent is related to the distance between the two opinions, that is, the smaller that distance, the larger the weight given to the comparison of the opinions. However, differently from [15], we assume that if the distance between the two opinions is larger than a given threshold, then there is no interaction and each agent does not change his own opinion anymore. Both aspects, i.e., proximity implying proportional weight and large distance implying absence of 
interaction, are considered, both from a theoretical and an empirical viewpoint, in [4], too.

Given this decisional mechanism, we tackle the issue of the evolution of the opinions over time, that is, whether they converge towards unanimity or if they give rise to other kinds of dynamical behaviors. In doing this, we introduce an auxiliary variable, describing the distance between the two agents' opinions. In such way, we are led to consider a one-dimensional dynamical system, for which we find a steady state in the origin, corresponding to the unanimity scenario, and a continuum of steady states related to the situation in which opinions are much different and thus agents do not interact and do not modify their opinions anymore. From a dynamical point of view, the only interesting steady state is the origin, given the instability of all other steady states. We find that an excessive reactivity destabilizes the unanimity fixed point through a first period-doubling bifurcation. A further increase in the reactivity parameter destabilizes the period-two cycle that, differently from the classical perioddoubling bifurcation scenario, gives rise, through a double pitchfork bifurcation of the second iterate, to two coexisting period-two cycles, that in turn bifurcate giving rise to a sequence of coexisting attractors of the same type until the emergence of chaos. Among the results above, we analytically investigate the stability of the unanimity steady state and the flip bifurcation through which it loses the stability, the double pitchfork bifurcation of the second iterate and the presence of chaotic dynamics. In particular, the existence of chaos is proved via the expander method in [7]. For the reader's convenience, we recall which concepts that technique is based on and we compare it with other well-known approaches in the chaos literature, explaining why, in the present framework, they are not convenient, or simply not working. In doing this, we also prove the existence of an absorbing interval for our dynamical system when the reactivity parameter is not too large.

On the other hand, due to the complexity of the computations involved, we show only numerically the subsequent period-doubling bifurcations of the coexisting periodic attractors leading to two coexisting periodic attractors, which then merge into a unique attractor, when increasing the reactivity parameter.

The remainder of the paper is organized as follows. In Section 2 we present our model. In Section 3 we perform the stability and bifurcation analysis, both analytically and numerically. In Section 4 we rigorously prove the presence of an absorbing interval and of complex dynamics, and we compare the technique we employ with other ones in the existing chaos literature. In Section 5 we discuss our results and draw some conclusions.

\section{The model}

Consider two agents, $A$ and $B$, between whom a decision formation process takes place. We suppose that each agent takes into account the decision of the other to a certain extent in forming his new own decision. This dynamic adaptive decision 
process can be repeated again and again and leads to a dynamical process in discrete time. In order to model the repeated process of decision formation, we take discrete time as a number periods $T=\{0,1,2, \ldots\}$. It is assumed that the decision of each agent is expressed by a real number, that is, the process is represented as a continuous decision dynamics, in contrast to the case of binary decision dynamics. For each agent $i \in\{A, B\}$, we denote his decision at time $t$ by $y_{i}(t)$. Thus, $y_{i}(t)$ is a real number and the bi-dimensional vector $y(t)=\left(y_{A}(t), y_{B}(t)\right)$ in $\mathbb{R}^{2}$ represents the decision profile at time $t$. Fixing an agent $i \in\{A, B\}$, the variation between his decisions at time $t+1$ and at time $t$ is proportional to the difference between his own decision and the decision of the other agent at time $t$ and to an endogenous positive reactivity, we denote by $\gamma_{i}(t)$. With this notation, decision formation of agent $i$ can be described by the following adaptive scheme

$$
y_{i}(t+1)=y_{i}(t)+\gamma_{i}(t)\left(y_{j}(t)-y_{i}(t)\right), i \neq j \in\{A, B\},
$$

where $\gamma_{i}(t)$ is an endogenous reactivity decreasing with the distance between the decisions of the two agents. More precisely, we assume that the agents are homogeneous in the reactivity, that is, $\gamma_{A}(t)=\gamma_{B}(t)=\gamma(t)$, with

$$
\gamma(t)=\left\{\begin{array}{lll}
\alpha-\beta\left|y_{A}(t)-y_{B}(t)\right|, & \text { for } & \left|y_{A}(t)-y_{B}(t)\right| \leq \frac{\alpha}{\beta} \\
0, & \text { for } & \left|y_{A}(t)-y_{B}(t)\right|>\frac{\alpha}{\beta}
\end{array}\right.
$$

where $\alpha$ and $\beta$ are positive constants.

Inserting $\gamma(t)$ from (2.2) into (2.1), we obtain the bi-dimensional system

$$
\left\{\begin{array}{l}
y_{A}(t+1)=y_{A}(t)+\gamma(t)\left(y_{B}(t)-y_{A}(t)\right) \\
y_{B}(t+1)=y_{B}(t)+\gamma(t)\left(y_{A}(t)-y_{B}(t)\right)
\end{array}\right.
$$

Introducing the auxiliary variable $z(t)=y_{A}(t)-y_{B}(t)$, representing the difference between the two decisions at time $t$, and subtracting the two equations in (2.3), we obtain

$$
z(t+1)= \begin{cases}z(t)-2 z(t)(\alpha-\beta|z(t)|), & \text { for }|z(t)| \leq \frac{\alpha}{\beta} \\ z(t), & \text { for }|z(t)|>\frac{\alpha}{\beta}\end{cases}
$$

that is,

$$
z(t+1)= \begin{cases}(1-2 \alpha) z(t)-2 \beta z(t)^{2}, & \text { for }-\frac{\alpha}{\beta} \leq z(t)<0 \\ (1-2 \alpha) z(t)+2 \beta z(t)^{2}, & \text { for } \frac{\alpha}{\beta} \geq z(t) \geq 0 \\ z(t), & \text { else }\end{cases}
$$


Through the change of variable ${ }^{1} x(t)=-\frac{2 \beta}{1-2 \alpha} z(t)$, we can rewrite (2.4) as follows

$$
x(t+1)= \begin{cases}(1-2 \alpha)\left(x(t)+x(t)^{2}\right), & \text { for } \frac{2 \alpha}{1-2 \alpha} \leq x(t)<0 \\ (1-2 \alpha)\left(x(t)-x(t)^{2}\right), & \text { for }-\frac{2 \alpha}{1-2 \alpha} \geq x(t) \geq 0 \\ x(t), & \text { else }\end{cases}
$$

Setting now $\mu=-(1-2 \alpha)$, we finally obtain

$$
x(t+1)= \begin{cases}-\mu\left(x(t)+x(t)^{2}\right), & \text { for }-\frac{\mu+1}{\mu} \leq x(t)<0 \\ -\mu\left(x(t)-x(t)^{2}\right), & \text { for } \frac{\mu+1}{\mu} \geq x(t) \geq 0 \\ x(t), & \text { else }\end{cases}
$$

with $\mu$ positive parameter (since $\alpha>\frac{1}{2}$ ), whose influence we will investigate in the next sections. Indeed, the dynamic equation in (2.6) is the one we are going to consider in the remainder of the paper, as, differently from the bi-dimensional formulation in (2.3), it can be studied analytically and still allows to get results that are easily interpretable. For instance, as we shall see in Section 3, the unique dynamically interesting steady state is the origin, which coincides with the conformism scenario. In view of the subsequent analysis, it is expedient to consider the one-dimensional map $f: \mathbb{R} \rightarrow \mathbb{R}$ related to (2.6), defined as

$$
f(x ; \mu)= \begin{cases}-\mu\left(x+x^{2}\right), & \text { for }-\frac{\mu+1}{\mu} \leq x<0 \\ -\mu\left(x-x^{2}\right), & \text { for } \frac{\mu+1}{\mu} \geq x \geq 0 \\ x, & \text { else }\end{cases}
$$

When comparing the dynamical system generated by the reactivity in (2.2) with the one analyzed in [15], some crucial differences emerge. Indeed, although the striking similarity between the shape of the graph of the maps generating the two dynamical systems, we stress that the map $f$ in (2.7) permits an analytical analysis, we are going to perform in the next sections, while the map considered in [15] is studied mainly numerically, because of the complexity of the computations involved. Moreover, as observed in the Introduction, also the decisional mechanisms leading to their formulations differ. In fact, in the present framework, the reactivity in (2.2) vanishes when the distance between the two opinions is large enough, while the reactivity considered in [15] is decreasing with the distance between the two opinions and tends to zero in the limit, but it never vanishes.

\footnotetext{
${ }^{1}$ In what follows, we will assume $\alpha>\frac{1}{2}$, so that $-\frac{2 \beta}{1-2 \alpha}$ is a positive quantity and thus there is no need to reverse inequalities when passing from (2.4) to (2.5). In fact, as it is easy to see, the case with $\alpha \leq \frac{1}{2}$ is not interesting from a dynamical viewpoint.
} 


\section{$3 \quad$ Stability and bifurcation analysis}

As a first step in the analysis of our system, in the next result we study the stability conditions for the fixed point $x=0$. There exist in fact also the continuum of symmetric fixed points $\left\{ \pm x: x \in\left[\frac{\mu+1}{\mu},+\infty\right)\right\}$ but they are always unstable and thus not interesting in view of the subsequent bifurcation analysis.

Proposition 3.1 For the map $f$ in (2.7) the fixed point $x=0$ is locally stable if and only if $\mu<1$.

Proof. The conclusion immediately follows by observing that for every $x$ in a sufficiently small neighborhood of 0 , it holds ${ }^{2}$ that

$$
\frac{\partial f}{\partial x}(x ; \mu)= \begin{cases}-2 \mu x-\mu, & \text { for } x \leq 0 \\ 2 \mu x-\mu, & \text { for } x \geq 0\end{cases}
$$

Thus $\frac{\partial f}{\partial x}(0 ; \mu)=-\mu \in(-1,1)$ if and only if $\mu<1$.

Hence, for $\mu<1$, the origin is a stable fixed point and it loses its stability for $\mu$ just above one. In Lemma 3.1 below we show that the stability is lost via a perioddoubling bifurcation. In the proof we will use the next result taken from [6], suitably rewritten consistently with our notation.

Theorem 3.1 (THEOREM 2.7, page 79, Elaydi (2000)) (Period-Doubling Bifurcation) If a one-dimensional smooth ${ }^{3}$ map $g: \mathbb{R} \rightarrow \mathbb{R}, x \mapsto g(x ; b)$, depending on the real parameter $b$, satisfies the following conditions:

(C1) $g\left(x^{*} ; b\right)=x^{*}$, for all $b$ in a neighborhood of $b^{*}$;

(C2) $\frac{\partial g}{\partial x}\left(x^{*} ; b^{*}\right)=-1$;

(C3) $\frac{\partial^{2} g^{2}}{\partial x \partial b}\left(x^{*} ; b^{*}\right) \neq 0$,

\footnotetext{
${ }^{2}$ Notice that the map $f$ is continuous on $\mathbb{R}$, it is $C^{1}$ on $\widehat{\mathbb{R}}:=\mathbb{R} \backslash\left\{ \pm \frac{\mu+1}{\mu}\right\}$ and $C^{2}$ on $\widehat{\mathbb{R}} \backslash\{0\}$. However, we will do not need to compute derivatives of order higher than one in the origin. As regards the fixed points $x= \pm \frac{\mu+1}{\mu}$, we stress that the left partial derivative $\frac{\partial f}{\partial x}{ }^{-}\left(\frac{\mu+1}{\mu} ; \mu\right)=\mu+2>2$, while the right partial derivative $\frac{\partial f}{\partial x}^{+}\left(\frac{\mu+1}{\mu} ; \mu\right)=1$, and, symmetrically, $\frac{\partial f}{\partial x}^{-}\left(-\frac{\mu+1}{\mu} ; \mu\right)=1$, while $\frac{\partial f}{\partial x}^{+}\left(-\frac{\mu+1}{\mu} ; \mu\right)=\mu+2>2$. For the fixed points $y \in\left\{ \pm x: x \in\left(\frac{\mu+1}{\mu},+\infty\right)\right\}$ it holds instead that $\frac{\partial f}{\partial x}(y ; \mu)=1$, for every $\mu$. Thus, as stated above, all fixed points but the origin are always unstable. ${ }^{3}$ Actually, the map $g$ needs to be smooth only in a neighborhood of $\left(x^{*} ; b^{*}\right)$, and the same is true in the statement of Theorem 3.2. In fact, the map $f$ in (2.7) is not differentiable for $x= \pm \frac{\mu+1}{\mu}$, but in Lemma 3.1 we are only interested in the behavior of $f$ and $f^{2}$ in a neighborhood of the origin, while in Proposition 3.2 we will only consider the behavior of $f^{2}$ in a neighborhood of $\tilde{x}=\frac{\mu-1}{\mu}$.
} 
then there exists an interval $I$ about $x^{*}$ and $a \mathcal{C}^{1}$ function $p: I \rightarrow \mathbb{R}$ such that $g(x ; p(x)) \neq x$ but $g^{2}(x ; p(x))=x$.

In what follows, we will also need the expression of the second iterate of $f$. As we shall see, its formulation slightly changes according to the value of $\mu$. Indeed, if the maximum value of $f$ exceeds the value of the fixed point $\frac{\mu+1}{\mu}$ (and, symmetrically, the minimum value of $f$ is below the value of the fixed point), which, as shown in Proposition 4.2 , happens for $\mu>2+\sqrt{8}$, then the expression of $f^{2}$ reads as follows:

$$
f^{2}(x ; \mu)= \begin{cases}\mu^{2} x(1+x)(1-\mu x(x+1)), & \text { for } x \in\left[-\frac{\mu+1}{\mu},-1\right] \\ \mu^{2} x(1+x)(1+\mu x(x+1)), & \text { for } x \in\left[-1,-x_{2}\right] \cup\left[-x_{1}, 0\right] \\ \mu x(-1-x), & \text { for } x \in\left[-x_{2},-x_{1}\right] \\ \mu^{2} x(1-x)(1+\mu x(x-1)), & \text { for } x \in\left[0, x_{1}\right] \cup\left[x_{2}, 1\right] \\ \mu x(x-1), & \text { for } x \in\left[x_{1}, x_{2}\right] \\ \mu^{2} x(x-1)(\mu x(x-1)-1), & \text { for } x \in\left[1, \frac{\mu+1}{\mu}\right] \\ x, & \text { else }\end{cases}
$$

where we denoted by $0<x_{1}<x_{2}<1$ the two solutions to the equation $\left.f(x)\right|_{[0,1]}=$ $-\frac{\mu+1}{\mu}$, so that, by symmetry, $-1<-x_{2}<-x_{1}<0$ are the two solutions to the equation $\left.f(x)\right|_{[-1,0]}=\frac{\mu+1}{\mu}$. We depict $x_{1}$ and $x_{2}$ in Figure 1 when $\mu=6$.

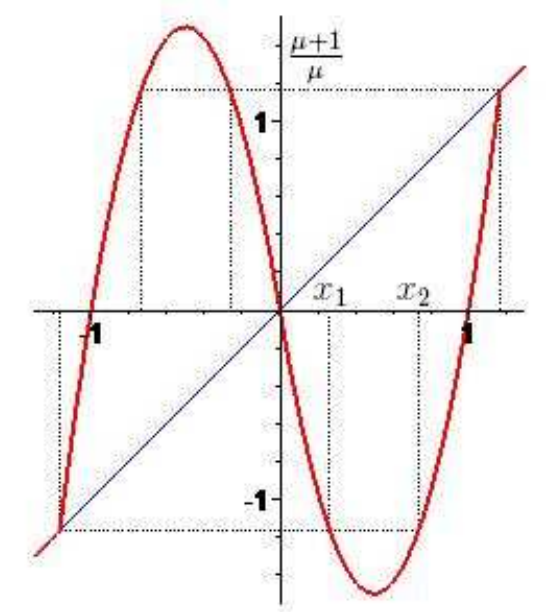

Figure 1: The map $f$ with $\mu=6$ and the solutions $x_{1}$ and $x_{2}$ to the equation $\left.f(x)\right|_{[0,1]}=-\frac{\mu+1}{\mu}$. 
If instead $\mu<2+\sqrt{8}$, we will show in Proposition 4.2 that there exists an absorbing interval and the expression of $f^{2}$ gets slightly simplified as follows:

$$
f^{2}(x ; \mu)= \begin{cases}\mu^{2} x(1+x)(1-\mu x(x+1)), & \text { for } x \in\left[-\frac{\mu+1}{\mu},-1\right] \\ \mu^{2} x(1+x)(1+\mu x(x+1)), & \text { for } x \in[-1,0] \\ \mu^{2} x(1-x)(1+\mu x(x-1)), & \text { for } x \in[0,1] \\ \mu^{2} x(x-1)(\mu x(x-1)-1), & \text { for } x \in\left[1, \frac{\mu+1}{\mu}\right] \\ x, & \text { else }\end{cases}
$$

Notice that, in the results below we will consider $1+\sqrt{6}$ as largest value for $\mu$ and thus only the formulation of $f^{2}$ in (3.2) will be used.

Lemma 3.1 For the map $f$ in (2.7) a period-doubling bifurcation occurs around $x=0$ when $\mu=1$.

Proof. In view of Theorem 3.1, we just have to check that conditions $(C 1),(C 2)$ and $(C 3)$ do hold true for $f$ when $(x ; \mu)=(0 ; 1)$.

Condition $(C 1)$ is trivially satisfied since $f(0 ; \mu)=0$, for all $\mu$.

As regards $(C 2)$, notice that $\frac{\partial f}{\partial x}(0 ; \mu)=-\mu$. Hence $\frac{\partial f}{\partial x}(0 ; 1)=-1$, as desired.

In order to check condition $(C 3)$, a direct computation starting from (3.2) shows that $\frac{\partial^{2} f^{2}}{\partial x \partial \mu}(0 ; \mu)=2 \mu$. Thus $\frac{\partial^{2} f^{2}}{\partial x \partial \mu}(0 ; 1)=2 \neq 0$. This concludes the proof.

In order to understand when new points of period two get born in the region $[-1,1]$, that is, the interesting area from a dynamical viewpoint ${ }^{4}$, let us consider $\left.f^{2}\right|_{[-1,1]}$. In fact, since the map $f$ is odd, and thus $f^{2}$ is odd, too, we will restrict our attention to $\left.f^{2}\right|_{[0,1]}$. The conclusions drawn there will then hold for $\left.f^{2}\right|_{[-1,0]}$ as well, just changing sign where needed.

Proposition 3.2 below gathers some information about the points of period two on $[0,1]$ and their stability. In particular, in order to show the presence of a pitchfork bifurcation, in the proof of Proposition 3.2 we will use the next well-known result (see $[21]$ ), we recall here for the reader's convenience:

Theorem 3.2 (Pitchfork Bifurcation) If a one-dimensional smooth map $g: \mathbb{R} \rightarrow \mathbb{R}$, $x \mapsto g(x ; b)$, depending on the real parameter $b$, satisfies the following conditions:

\footnotetext{
${ }^{4}$ Simple calculations show indeed that $x=\frac{\mu+1}{\mu}$, i.e., a fixed point, is the only point of period two for $f$ on $\left(1, \frac{\mu+1}{\mu}\right]$, and similarly $x=-\frac{\mu+1}{\mu}$ is the only point of period two for $f$ on $\left[-\frac{\mu+1}{\mu},-1\right)$. As already remarked, all points in $\left(-\infty,-\frac{\mu+1}{\mu}\right) \cup\left(\frac{\mu+1}{\mu}, \infty\right)$ are fixed points, too.
} 


$$
\begin{array}{lll}
\left(C 1^{\prime}\right) & g\left(x^{*} ; b^{*}\right)=x^{*} ; \\
\left(C 2^{\prime}\right) & \frac{\partial g}{\partial x}\left(x^{*} ; b^{*}\right)=1 ; \\
\left(C 3^{\prime}\right) \quad & \frac{\partial g}{\partial b}\left(x^{*} ; b^{*}\right)=0, & \frac{\partial^{2} g}{\partial x^{2}}\left(x^{*} ; b^{*}\right)=0, \\
& \frac{\partial^{2} g}{\partial x \partial b}\left(x^{*} ; b^{*}\right) \neq 0, & \frac{\partial^{3} g}{\partial x^{3}}\left(x^{*} ; b^{*}\right) \neq 0,
\end{array}
$$

then a pitchfork bifurcation for $g$ occurs at $\left(x^{*} ; b^{*}\right)$.

Proposition 3.2 In addition to the fixed point $x=0$, the points of period two for $f$ on $[0,1]$ are:

- for $\mu>1, \tilde{x}=\frac{\mu-1}{\mu}$;

- for $\mu>3, \bar{x}=\frac{(\mu+1)-\sqrt{\mu^{2}-2 \mu-3}}{2 \mu}$ and $\overline{\bar{x}}=\frac{(\mu+1)+\sqrt{\mu^{2}-2 \mu-3}}{2 \mu}$.

In particular, $\tilde{x}$ gets originated when the origin has the period-doubling bifurcation for $\mu=1$ and $\tilde{x}$ is stable for $1<\mu<3$. For $\mu=3, \tilde{x}$ undergoes a pitchfork bifurcation and $\bar{x}$ and $\overline{\bar{x}}$ get born, which are stable for $3<\mu<1+\sqrt{6}$.

Proof. The points of period two $\tilde{x}, \bar{x}$ and $\overline{\bar{x}}$ are found just imposing the condition $\left.f^{2}(x ; \mu)\right|_{[0,1]}=\mu^{2} x(1-x)(1+\mu x(x-1))=x \in[0,1]$. As usual, the stability conditions are determined by setting $\frac{\partial f^{2}}{\partial x}(\hat{x}) \in(-1,1)$, where $\hat{x}$ is one of the periodic points found above. The period-doubling bifurcation of the origin has been proven in Lemma 3.1, while the pitchfork bifurcation of $\tilde{x}$ can be shown by checking that the conditions $\left(C 1^{\prime}\right),\left(C 2^{\prime}\right)$ and $\left(C 3^{\prime}\right)$ in Theorem 3.2 hold for $f^{2}$ when $(x, \mu)=(\tilde{x}, 3)$. In fact, as regards $\left(C 1^{\prime}\right)$, a direct computation shows that $f^{2}(\tilde{x} ; \mu)=\tilde{x}$, for every $\mu>1$. In regard to $\left(C 2^{\prime}\right)$, it is easy to prove that, for $\mu>1, \frac{\partial f^{2}}{\partial x}(\tilde{x} ; \mu)=1$ if and only if $\mu=3$. As concerns $\left(C 3^{\prime}\right)$, it holds that, for $\mu>1, \frac{\partial f^{2}}{\partial \mu}(\tilde{x} ; \mu)=\frac{(3-\mu)(\mu-1)}{\mu^{2}}=0$ if and only if $\mu=3$. Moreover, $\frac{\partial^{2} f^{2}}{\partial x^{2}}(\tilde{x} ; \mu)=2 \mu\left(-\mu^{2}+5 \mu-6\right)=0$ for $\mu=3$ and $\frac{\partial^{2} f^{2}}{\partial x \partial \mu}(\tilde{x} ; 3)=2 \neq 0$. Finally, $\frac{\partial^{3} f^{2}}{\partial x^{3}}(\tilde{x} ; 3)=-108 \neq 0$. This concludes the proof.

In regard to the points of period two on $[0,1]$ found in Proposition 3.2 and their symmetric counterpart on $[-1,0]$, it is easy to check that

$$
\begin{aligned}
f\left(\frac{\mu-1}{\mu} ; \mu\right) & =\frac{1-\mu}{\mu}, \\
f\left(\frac{(\mu+1)-\sqrt{\mu^{2}-2 \mu-3}}{2 \mu} ; \mu\right) & =\frac{-(\mu+1)-\sqrt{\mu^{2}-2 \mu-3}}{2 \mu}
\end{aligned}
$$


and

$$
f\left(\frac{(\mu+1)+\sqrt{\mu^{2}-2 \mu-3}}{2 \mu} ; \mu\right)=\frac{-(\mu+1)+\sqrt{\mu^{2}-2 \mu-3}}{2 \mu} .
$$

From the previous results, it looks evident that an increasing value for $\mu$ makes the behavior of the map $f$ more and more complex, with the loss of stability of the previously existing periodic points and the emergence of new periodic orbits. We illustrate this phenomenon in Figures 2-6 below. In particular, in Figure 2 the parameter $\mu$ is set equal to 0.5 and thus, according to Proposition 3.1, the unique fixed point, i.e., the origin, is locally stable. In Figures 3 and 4 , where we set $\mu=2$, according to Lemma 3.1, the origin has lost its stability via a flip bifurcation and, according to Proposition 3.2, the points of period two $\tilde{x}=\frac{1}{2}$ and its symmetric counterpart $-\tilde{x}$ have emerged. In Figure 5 we increase the parameter $\mu$ to 3.3 , so that, according to Proposition 3.1, the two periodic points $\pm \tilde{x}= \pm 0 . \overline{69}$ lose their stability via a double pitchfork bifurcation and the points of period two $\bar{x}=0.479$ and $\overline{\bar{x}}=0.823$ and their symmetric counterparts $-\bar{x}$ and $-\overline{\bar{x}}$ emerge. In Figure 6 we set $\mu=3.7>1+\sqrt{6}$ and thus, according to Proposition 3.2 , also $\pm \bar{x}= \pm 0.39$ and $\pm \overline{\bar{x}}= \pm 0.88$ are no more stable.

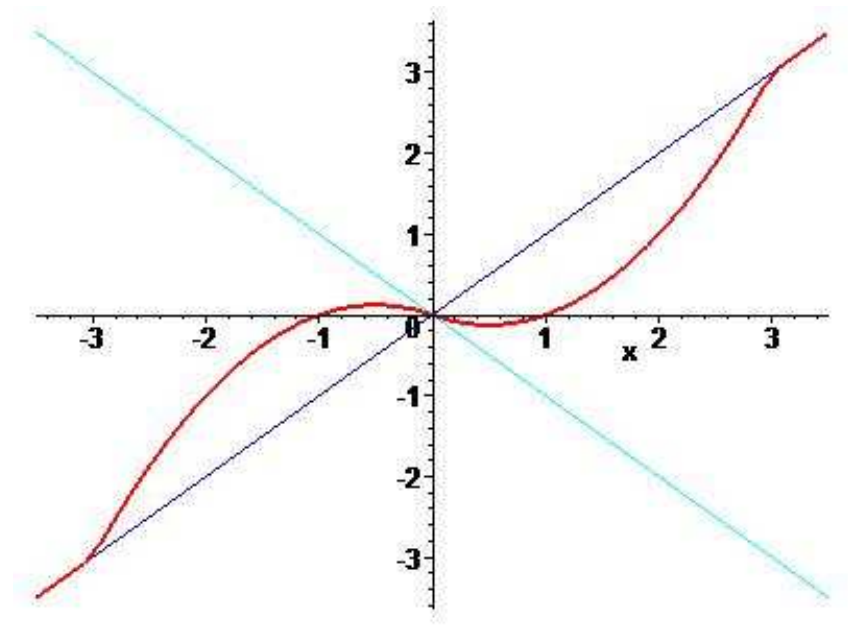

Figure 2: The map $f$ with $\mu=0.5$.

When comparing the process described above with what happens to the logistic map when increasing the corresponding parameter, in addition to several similarities, we also remark a crucial difference. In fact, instead of undergoing the standard period-doubling route to chaos characteristic of the logistic map, we observe for the map $f$ in (2.7) that when the first orbit of period two becomes unstable, two new cycles of period two, coexisting with the old one, appear through a double pitchfork bifurcation of $f^{2}$. Such new cycles are stable at the beginning, but increasing $\mu$ they lose stability via period-doubling bifurcations leading to two coexisting cycles of period four, which again become unstable for larger values of $\mu$. 


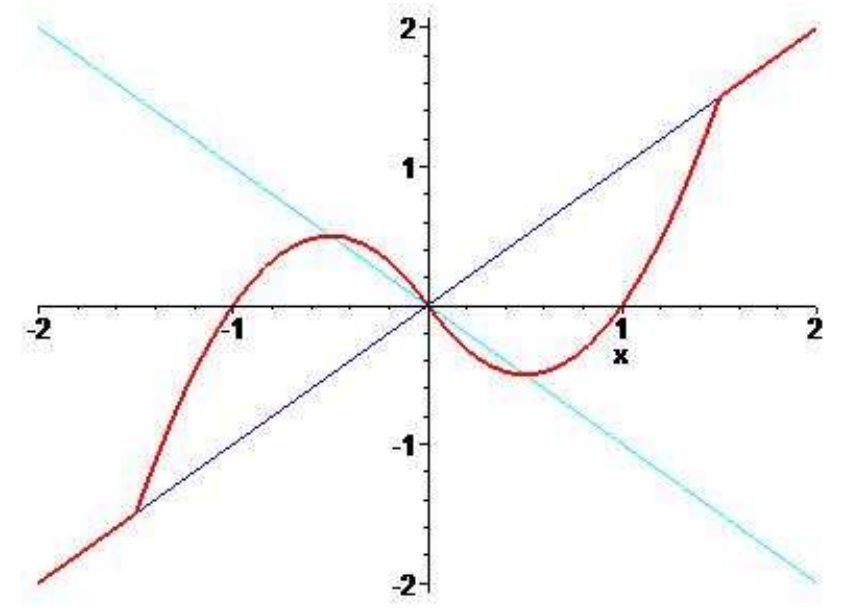

Figure 3: The map $f$ with $\mu=2$.

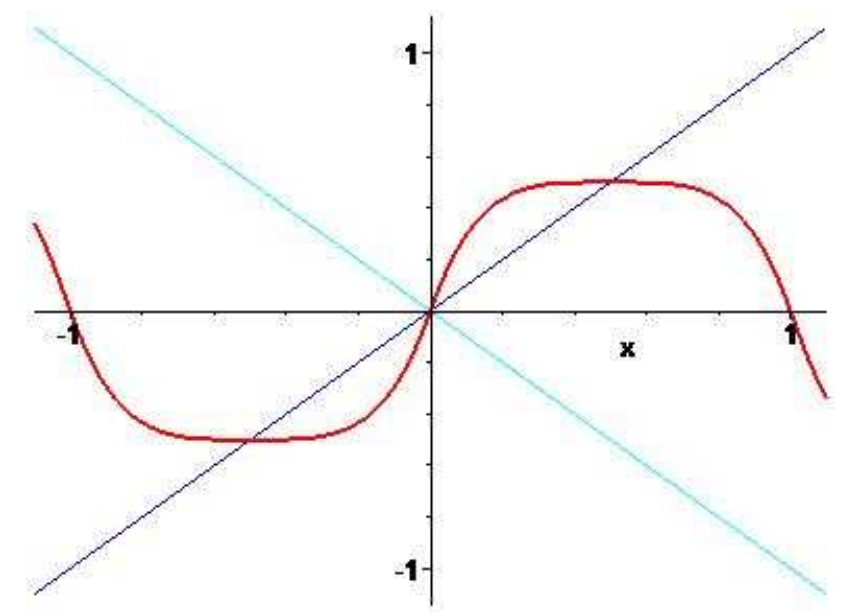

Figure 4: The map $f^{2}$ with $\mu=2$.

Due to the difficulty in computing the forth iterate of the map $f$, we omit the analytical treatment of these steps and in the next section we directly prove the existence of chaotic dynamics. For the reader's convenience, we however present in Figure 7 the bifurcation diagram of $f$ with respect to $\mu$, which illustrates the steps above and also shows that the chaotic regime is interrupted by some periodicity windows. We stress that there is a perfect agreement between the theoretical results and the first bifurcation values in Figure 7. In particular, we observe the pitchfork bifurcation of the period-two cycle when $\mu=3$ and the subsequent emergence of two coexisting period-two cycles, which in turn bifurcate when $\mu=1+\sqrt{6} \approx 3.45$ giving rise to two coexisting period-four cycles. The latter bifurcate for $\mu \approx 3.545$ generating two coexisting period-eight cycles, which in turn give rise to two coexisting chaotic attractors in eight pieces when $\mu \approx 3.574$, generating two coexisting chaotic attractors 


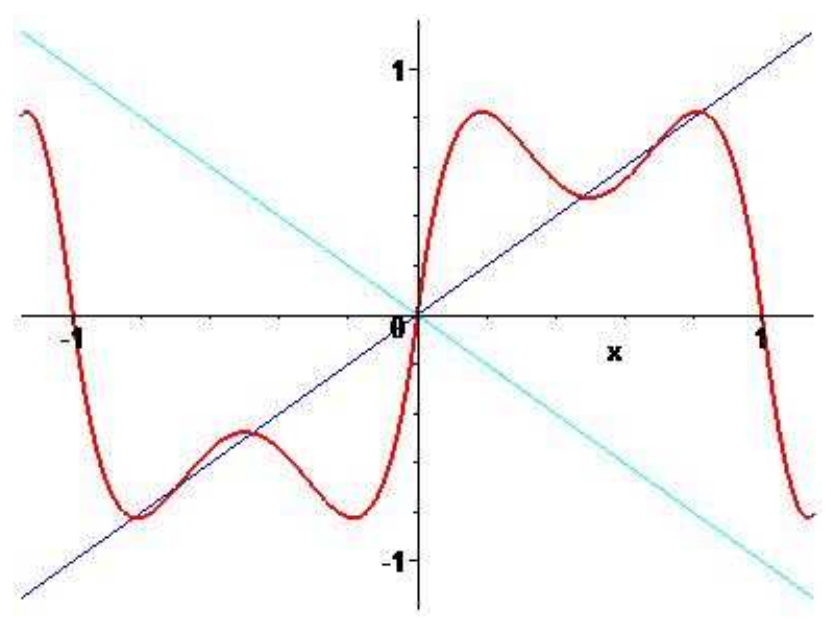

Figure 5: The map $f^{2}$ with $\mu=3.3$.

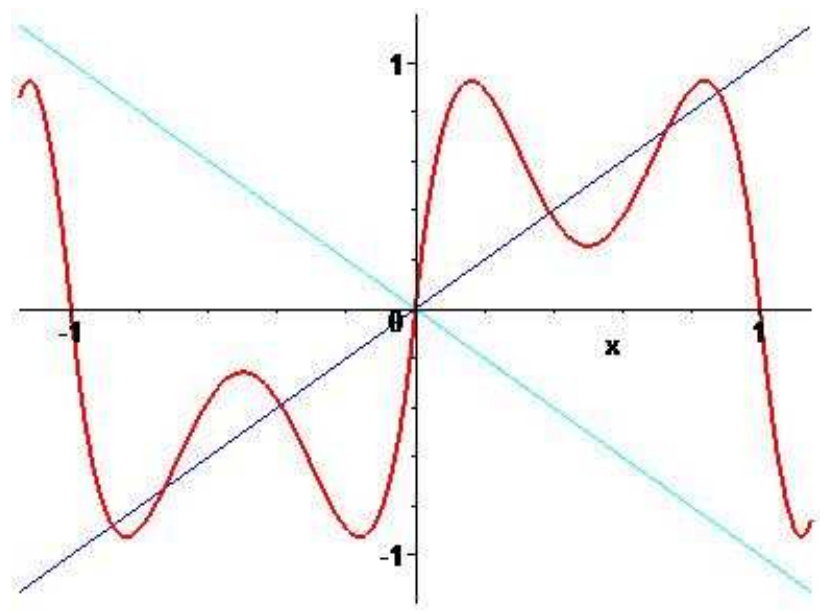

Figure 6: The map $f^{2}$ with $\mu=3.7$.

in four pieces when $\mu \approx 3.576$. Those give rise to two coexisting chaotic attractors in two pieces when $\mu \approx 3.593$, which generate a unique chaotic attractor in two pieces when $\mu \approx 3.673$, which in turn generates a unique chaotic attractor in one piece when $\mu=4$. A periodicity window can be observed for $\mu \approx 3.82$. As we shall see in Proposition 4.2 below, for $\mu<2+\sqrt{8}$, there exists an absorbing interval $I \subset\left(-\frac{\mu+1}{\mu}, \frac{\mu+1}{\mu}\right)$. When however $\mu$ exceeds that threshold, then, for any initial condition, the iterates of the map $f$ will eventually hit the set $\left(-\infty,-\frac{\mu+1}{\mu}\right] \cup\left[\frac{\mu+1}{\mu},+\infty\right)$, entirely composed of fixed points. This is the reason why in Figure 7 for $\mu>2+\sqrt{8} \approx 4.828$ the chaotic band stops and two segments appear in its place. 


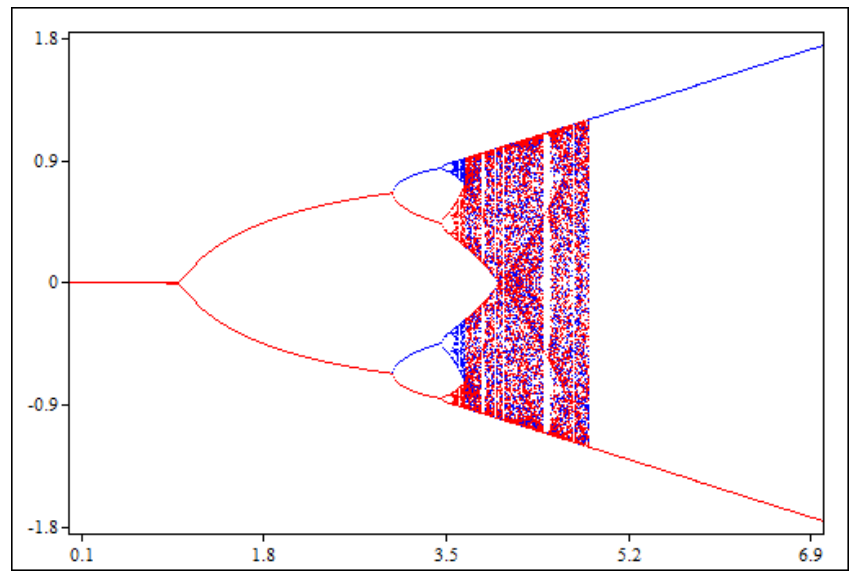

Figure 7: The bifurcation diagram for $f$ w.r.t. $\mu \in[0,7]$. The blue dots are obtained for the initial condition $x(0)=0.5$, while the red dots are obtained for the initial condition $x(0)=-0.5$.

\section{Chaotic dynamics}

In order to prove the presence of chaotic dynamics for our system, we apply the expander method from [7]. More specifically, in Proposition 4.1 below we will use the Chaos Lemma in [7]. With this respect, we recall hereinafter some useful definitions. We warn the reader that sometimes we will replace the general setting in [7] with the specific framework we are considering.

Let $Q$ be a metric space, $Q_{0}$ be a compact subset of $Q$ and $\varphi: Q_{0} \rightarrow Q$ be a continuous map. Let $S_{1}$ and $S_{2}$ be nonempty, compact and disjoint subsets of $Q_{0}$ and let $\mathcal{E}$ be a nonempty family of nonempty subsets of $Q$ such that, for each $E \in \mathcal{E}$ and each $S_{i}, i \in\{1,2\}$, there exists a compact set $P_{i} \subset E \cap S_{i}$ such that $\varphi\left(P_{i}\right) \in \mathcal{E}$.

The family $\mathcal{E}$ is called family of expanders in [7] and each $E \in \mathcal{E}$ is called expander. The Chaos Lemma (cf. [7, Lemma 4]) states that if the assumptions above are fulfilled, then there exists a compact, invariant set $Q_{*} \subset Q_{0}$ such that the map $\varphi$ displays on it sensitive dependence with respect to initial conditions and each sequence on two symbols is followed by the $\varphi$-orbit of a point in $Q_{*}$. Actually, from the proof of the Chaos Lemma in [7], it also follows that the map $\varphi$ is topologically transitive on $Q_{*}$. In order to explain this step, we need to recall some further definitions. We name dynamical systems the pairs $(Z, l)$, where $Z$ is a compact metric space and $l: Z \rightarrow Z$ is continuous and onto. Given $z \in Z$, we call the set $\gamma(z):=\left\{l^{n}(z): n \in \mathbb{N}\right\}$ the forward $l$-orbit of $z$ and we denote by $\overline{\gamma(z)}$ its closure. Given $z \in Z$, we define the $\omega$-limit set of $z$ as $\omega(z):=\left\{x \in Z: \exists n_{j} \nearrow+\infty\right.$ with $\left.l^{n_{j}}(z) \rightarrow x\right\}$. According to [20, Theorem 5.5], $\omega(z)$ is closed, nonempty and invariant by the compactness of $Z$. Moreover it holds that $\overline{\gamma(z)}=\gamma(z) \cup \omega(z)$.

Turning back to the proof of [7, Lemma 4], therein it is shown that there exists $x^{*} \in Q_{0}$ such that $x^{*} \in \omega\left(x^{*}\right)$ and, by construction, $Q_{*}=\omega\left(x^{*}\right)$. Since $x^{*} \in \omega\left(x^{*}\right)$, 
by the invariance of the $\omega$-limit sets, also all iterates of $x^{*}$ belong to $\omega\left(x^{*}\right)$, and thus $\gamma\left(x^{*}\right) \subset \omega\left(x^{*}\right)$. Hence $\overline{\gamma\left(x^{*}\right)}=\gamma\left(x^{*}\right) \cup \omega\left(x^{*}\right)=\omega\left(x^{*}\right)=Q_{*}$ and then the forward $\varphi$-orbit of $x^{*}$ is dense in $Q_{*}$. On the other hand, by the invariance of $Q_{*}$, the pair $\left(Q_{*},\left.\varphi\right|_{Q_{*}}\right)$ is a dynamical system and, according to [1, Lemma 3], in every dynamical system the existence of a dense orbit is equivalent to the topological transitivity of the associated map.

In order to apply the Chaos Lemma to prove the presence of a chaotic set in our context, in what follows we will consider $\mu>4$ and take ${ }^{5}$

$$
\begin{gathered}
Q=\mathbb{R}, \quad Q_{0}=[-1,1], \quad \varphi=\left.f\right|_{[-1,1]}, \\
S_{1}=\left[-1, \frac{-\mu-\sqrt{\mu^{2}-4 \mu}}{2 \mu}\right] \cup\left[\frac{\mu+\sqrt{\mu^{2}-4 \mu}}{2 \mu}, 1\right], \\
S_{2}=\left[\frac{-\mu+\sqrt{\mu^{2}-4 \mu}}{2 \mu}, \frac{\mu-\sqrt{\mu^{2}-4 \mu}}{2 \mu}\right], \\
\mathcal{E}=\{E\}=\{[-1,1]\}, \quad P_{i}=E \cap S_{i}=S_{i}, i \in\{1,2\} .
\end{gathered}
$$

See Figure 8 for a graphical illustration of the sets $S_{1}$ and $S_{2}$ when $\mu=4.5$. Observe that, with the choices above, it holds that $\varphi\left(P_{i}\right)=E, i \in\{1,2\}$, and thus the Chaos Lemma in [7] allows us to infer the existence of a chaotic set in $[-1,1]$. We summarize our findings in the next result.

Proposition 4.1 Let $\mu>4$. Then there exists a compact, invariant set $I^{*} \subset[-1,1]$ such that the map $f$ displays on it sensitive dependence with respect to initial conditions, topological transitivity and each sequence on two symbols is followed by the $f$-orbit of a point in $I^{*}$.

We stress that we chose to employ the expander method in [7] because in the present framework the other approaches in the chaos literature we know do not allow to prove the presence of a complex behavior or would lead to cumbersome computations. For instance, an attempt would be that of trying to apply the concept of covering intervals in [3] plus some tools from the theory of symbolic dynamics in $[9,11]$ in order to show that the map $f$ has positive topological entropy, i.e., one of the trademarks of chaos. Another attempt would consist in using the "stretching along the paths" (from now on, SAP) method, already employed, for instance, in [13] and [18] to prove the existence of chaotic dynamics for both discrete and continuous-time dynamical systems. A third approach would then consist in working with the first three iterates of the map $f$ in (2.7), in order to find suitable intervals where to apply Theorem 1 in [10] so as to prove the existence of chaos in the sense of Li-Yorke, as

\footnotetext{
${ }^{5}$ Notice that the extreme values for $S_{1}$ and $S_{2}$ depending on $\mu$ are found by setting $\left.f\right|_{[-1,0]}=1$ and $\left.f\right|_{[0,1]}=-1$. They are well defined for $\mu>4$.
} 


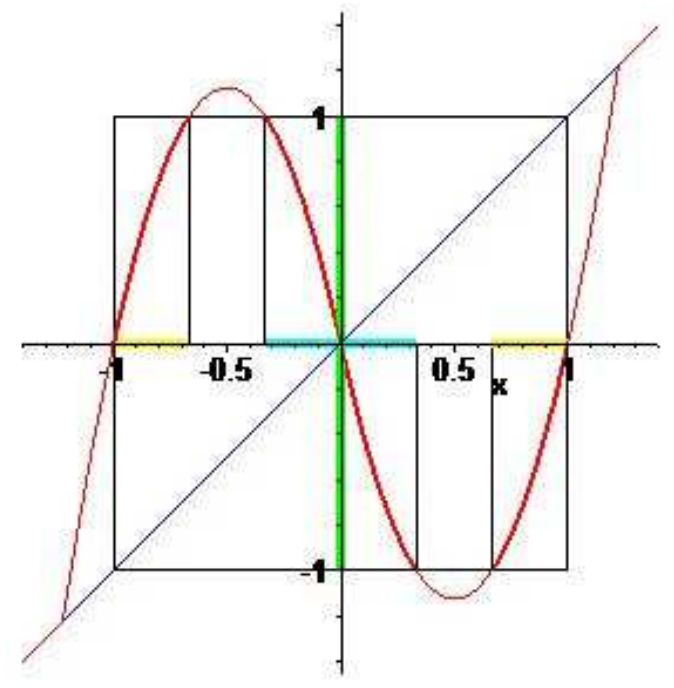

Figure 8: A graphical illustration of the construction behind Proposition 4.1 for $f$ with $\mu=4.5$. In particular, the yellow and cyan sets on the $x$-axis are $S_{1}$ and $S_{2}$ in (4.2) and (4.3), respectively.

described in conditions (T1) and (T2) in that result. This method has recently been used to rigorously prove the presence of chaotic dynamics in [15] (see [15, Proposition $4.2]$ ). Due to the similarity between the geometry of the present framework and the one in [15] we strongly believe that technique should work in the present framework as well. However, it requires to work even with the third iterate of the map $f$, whose expression here is not quick to compute and to handle. For this reason we found more convenient to rather apply the expander method in [7].

Let us now turn back to the covering interval approach and the SAP method, in order to explain why they seem not applicable in our framework.

As concerns the covering interval approach, we recall that, given a continuous mapping $g: \mathbb{R} \rightarrow \mathbb{R}$ and two intervals $I, J \subseteq \mathbb{R}$, we say that $I g$-covers $J$ if $g(I) \supseteq J$, or equivalently, if there exists a sub-interval $I_{0} \subseteq I$ such that $g\left(I_{0}\right)=J$. Moreover, given a continuous map $g: I \rightarrow I$ defined on a compact interval $I \subset \mathbb{R}$ and $n \geq 2$ closed sub-intervals $I_{0}, \ldots, I_{n-1} \subseteq I$, with pairwise disjoint interiors, we associate to the dynamical system $(I, g)$ the $n \times n$ transition matrix $T=T(j, k)$, for $j, k \in\{0, \ldots, n-1\}$, defined as

$$
T(j, k)= \begin{cases}1 & \text { if } I_{j} g \text {-covers } I_{k} \\ 0 & \text { else }\end{cases}
$$

It is possible to prove (cf. [9, Observation 1.4.2]) that, when $T$ is irreducible, i.e., for every couple of integers $j, k \in\{0, \ldots, n-1\}$ there exists a positive integer $\ell$ such that $T^{\ell}(j, k)>0$, then $h_{\text {top }}(g) \geq \log (\bar{\lambda})$, where $\bar{\lambda}$ is the largest real eigenvalue of $T$ in absolute value, also called Perron eigenvalue of $T$.

As a first step towards the application of the just explained strategy, we need to find 
a compact invariant interval for our map $f$ in (2.7). In the next result we prove that, under certain conditions on the parameter $\mu$, there exists an absorbing interval, which in particular is invariant.

Proposition 4.2 Call $m=\frac{1}{2}$ and $M=-\frac{1}{2}$ the unique local minimum point and local maximum point of the map $f$, respectively, and set $m^{\prime}:=f(m)$ and $M^{\prime}:=f(M)$. If $\mu<2+\sqrt{8}$, the compact interval $I=\left[m^{\prime}, M^{\prime}\right]$ is absorbing, i.e., there exists a compact interval $J \supset I$ such that, for all $\bar{x} \in J$ there exists $\bar{n} \in \mathbb{N}$ such that $f^{\bar{n}}(\bar{x}) \in I$ and for any $x \in I, f^{n}(x) \in I$, for all $n \in \mathbb{N}$.

Proof. We claim that the interval $J$ has as extreme points the fixed points $P_{1}=-\frac{\mu+1}{\mu}$ and $P_{2}=\frac{\mu+1}{\mu}$, respectively.

Let us show at first that $J \supset I$. A simple calculation shows that, since $\mu<2+\sqrt{8}$, then

$$
P_{1}=-\frac{\mu+1}{\mu}<m^{\prime}=-\frac{\mu}{4}<M^{\prime}=\frac{\mu}{4}<\frac{\mu+1}{\mu}=P_{2},
$$

as desired.

Let us then consider a generic starting point $\hat{x}$ in $J$ and show that its trajectory will eventually remain in $I$.

If $\hat{x} \in I$, then by construction its forward $f$-orbit will be trapped inside $I$, as well. In fact, since by continuity $f(x)<x$, for every $0<x<P_{2}$, and, for the considered parameter values, it holds that $M^{\prime}<P_{2}$, then it follows that $f\left(M^{\prime}\right)<M^{\prime}$ and thus $f\left(M^{\prime}\right) \in I$. A completely symmetric argument shows that when $\mu<2+\sqrt{8}$, then $f\left(m^{\prime}\right)>m^{\prime}$ and thus $f\left(m^{\prime}\right) \in I$, too.

Let us now analyze the two remaining cases, i.e., $P_{1}<\hat{x}<m^{\prime}$ and $P_{2}>\hat{x}>M^{\prime}$. Since $0 \in I$ and by continuity $f(x)>x$, for every $P_{1}<x<0$, and $f(x)<x$, for every $0<x<P_{2}$, if $P_{1}<\hat{x}<m^{\prime}$, then its iterates will approach $I$ in a strictly increasing way, while if $P_{2}>\hat{x}>M^{\prime}$, then its iterates will approach $I$ in a strictly decreasing way. Once that a forward iterate of $\hat{x}$ lies in $I$, then by construction all its subsequent iterates will be trapped inside $I$, as well. This concludes the proof.

A graphical illustration of the absorbing interval $I$ can be found in Figure 9 for $f$ with $\mu=4.5<2+\sqrt{8}$.

Notice that the threshold for the existence of an absorbing interval is higher than the threshold found in Proposition 4.1 for the existence of chaos. Hence, when $\mu \in$ $(4,2+\sqrt{8})$ both conditions are satisfied and thus both features are present in our dynamical system.

Hence, thanks to Proposition 4.2, when $\mu<2+\sqrt{8}$ the invariant compact interval we need in the covering interval strategy is the absorbing interval $I$. Moreover, for $\mu>4$ the natural choice for the covering intervals is given by $I_{0}=\left[-1, \frac{-\mu-\sqrt{\mu^{2}-4 \mu}}{2 \mu}\right]$, $I_{1}=\left[\frac{-\mu+\sqrt{\mu^{2}-4 \mu}}{2 \mu}, \frac{\mu-\sqrt{\mu^{2}-4 \mu}}{2 \mu}\right]$ and $I_{2}=\left[\frac{\mu+\sqrt{\mu^{2}-4 \mu}}{2 \mu}, 1\right]$, whose extreme values are 


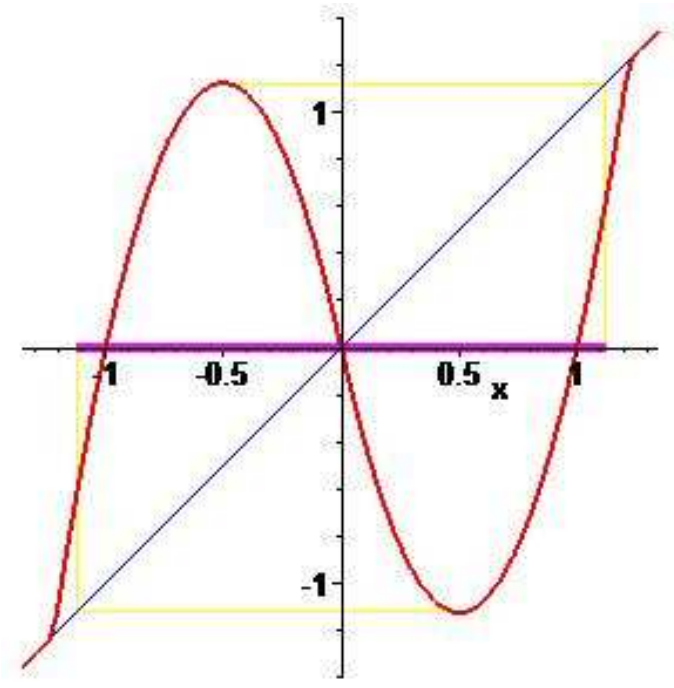

Figure 9: The highlighted set on the $x$-axis is the absorbing interval $I$ from Proposition 4.2 .

zeros of the map $f$ or the solutions to the equation $|f(x)|=1$. Notice that $I_{0}, I_{1}$ and $I_{2}$ are the three intervals highlighted on the $x$-axis in Figure 8. The corresponding transition matrix turns out to be

$$
T_{f}=\left(\begin{array}{lll}
0 & 0 & 1 \\
1 & 1 & 1 \\
1 & 0 & 0
\end{array}\right)
$$

and a simple calculation shows that it is not irreducible as $T_{f}^{n}=\left(\begin{array}{ccc}0 & 0 & 1 \\ n & 1 & n \\ 1 & 0 & 0\end{array}\right)$ when $n$ is odd and $T_{f}^{n}=\left(\begin{array}{ccc}1 & 0 & 0 \\ n & 1 & n \\ 0 & 0 & 1\end{array}\right)$ when $n$ is even. Thus the covering interval approach does not allow to draw any conclusions on the existence of chaos, at least for the choice of the covering intervals above.

Somewhat similarly, also the SAP method seems to be not applicable in view of proving the presence of complex dynamics in our framework. In fact, given a compact interval $I$ on which the map $\varphi$ we are analyzing is continuous, that technique in the one-dimensional setting requires to find (at least) two disjoint compact sub-intervals $I_{0}$ and $I_{1}$ of $I$ such that their $\varphi$-images cover $I$. In our context, however, taking $I=[-1,1]$, if $\mu<4$ then no such sub-intervals exist for the map $f$, while if $\mu>4$, only one such sub-interval exists, which can be chosen in several ways. The smallest suitable one is $S_{2}$ in (4.3), the largest is $I$ itself; any interval in between works as well. However, as it is easy to check, it is not possible to find two disjoint compact intervals as required by the SAP method, no matter what the value of $\mu$ is. Notice 
indeed that the $f$-image of the set $S_{1}$ in (4.2) covers $I$, but $S_{1}$ is not an interval.

This simple example illustrates that, when compared to the expander method in [7], the SAP technique requires in general stricter conditions in order to be applied. On the other hand, the latter allows to draw stronger conclusions from a dynamical viewpoint, as, for instance, the existence of fixed points is ensured and, in fact, the periodic orbits are dense in a suitable invariant set. The technique in [7] is instead not able to guarantee the existence of fixed points, nor periodic points of any period, as shown in [8, Example 10] or in [16, Figure 1.5], which is inspired to the bulging horseshoe in [7, Figure 4]. Nonetheless, as seen in Proposition 4.1, the method in [7] still allows to draw some interesting conclusions from a dynamical viewpoint. For a more detailed comparison between the covering relations in [7] and [13], we refer the interested reader to [17].

\section{Conclusion}

In this paper we proposed a framework to analyze the dynamical process of decision and opinion formation of two economic homogeneous and boundedly rational agents that interact and learn from each other over time. We did not consider intrinsic utility coming from economic and social activities; we considered instead only external influences on the decisional process. We adopted such a point of view in order to highlight the role of social interaction on that process. The decisional process presented in our model is an adaptive adjustment mechanism in which an agent takes into account the difference between his own opinion and the opinion of the other agent. The smaller that difference, the larger the weight put on the comparison of the opinions. We also assumed that if the distance between the two opinions is larger than a given threshold, then there is no interaction and the agents do not change their opinions anymore.

Introducing an auxiliary variable describing the distance between the opinions, we obtained a one-dimensional dynamical system for which we found a steady state in the origin, corresponding to the unanimity scenario, and a continuum of steady states related to the situation of absence of interaction. Analytically, we investigated the stability of the unanimity steady state, the first flip bifurcation through which it loses the stability and the double pitchfork bifurcation of the second iterate, as well as the presence of chaotic dynamics. Numerically, we showed the subsequent perioddoubling bifurcations of the coexisting periodic attractors leading to two coexisting periodic attractors, which then merge into a unique attractor, when increasing the reactivity parameter.

Future research should focus, for instance, on the extension of the model to the framework of three interacting agents. In this case, however, it seems in general not possible to reduce the dimensionality of the problem by introducing some auxiliary variables and thus the analytical tractability of the model gets drastically reduced.

Another interesting extension would concern the context of heterogeneous agents: in such case it would still be possible to obtain a one-dimensional model through a 
change of variable, but the resulting model would be heavier to analyze than that studied in the present paper.

We finally stress that models of the kind here presented are also suitable to be applied, for instance, in the context of financial asset markets with boundedly rational agents that ground their buying and selling decisions on fundamental values, which can be continuously updated on the basis of a comparative process among agents.

\section{References}

[1] J. Auslander, J.A. Yorke, Interval maps, factors of maps, and chaos, Tôhoku Math. J. 32 (1980) 177-188.

[2] M. Bianchi (ed.), The Active Consumer: Novelty and Surprise in Consumer Choice, Routledge, London, 1998.

[3] L. Block, J. Guckenheimer, M. Misiurewicz, L.S. Young, Periodic points and topological entropy of one-dimensional maps, in: Global theory of dynamical systems (Proc. Internat. Conf., Northwestern Univ., Evanston, Ill., 1979), Lecture Notes in Math. 819, Springer, Berlin, 1980, pp. 18-34.

[4] P.M. DeMarzo, D. Vayanos, J. Zwiebel, Persuasion bias, social influence and unidimensional opinions, Quarterly Journal of Economics 118 (2003) 909-968.

[5] J.-G. Du, T. Huang, Z. Sheng, Analysis of decision-making in economic chaos control, Nonlinear Analysis: Real World Applications 10 (2009) 2493-2501.

[6] S.N. Elaydi, Discrete Chaos, Chapman \& Hall/CRC, Boca Raton, 2000.

[7] J. Kennedy, S. Koçak, J.A. Yorke, A chaos lemma, Amer. Math. Monthly 108 (2001) 411-423.

[8] J. Kennedy, J.A. Yorke, Topological horseshoes, Trans. Amer. Math. Soc. 353 (2001) 2513-2530.

[9] B.P. Kitchens, Symbolic Dynamics. One-Sided, Two-Sided and Countable State Markov Shifts, Universitext. Springer-Verlag, Berlin, 1998.

[10] T.Y. Li, J.A. Yorke, Period three implies chaos, Amer. Math. Monthly 82 (1975) 985-992.

[11] D. Lind, B. Marcus, An Introduction to Symbolic Dynamics and Coding, Cambridge University Press, Cambridge, 1995. 
[12] A. Matsumoto, F. Szidarovszky, Delayed dynamics in heterogeneous competition with product differentiation, Nonlinear Analysis: Real World Applications 11 (2010) 601-611.

[13] A. Medio, M. Pireddu, F. Zanolin, Chaotic dynamics for maps in one and two dimensions. A geometrical method and applications to economics, Internat. J. Bifur. Chaos Appl. Sci. Engrg. 19 (2009) 3283-3309.

[14] S. Metcalfe, The economic foundation of technology policy: equilibrium and evolutionary perspectives, in: P. Stoneman (Ed.), Handbook of the Economics of Innovation and Technological Change, Blackwell, Oxford, 1995.

[15] A. Naimzada, M. Pireddu, Adaptive decision dynamics, submitted.

[16] M. Pireddu, Fixed points and chaotic dynamics for expansive-contractive maps in Euclidean spaces, with some applications. PhD thesis. Available at http://arxiv.org/pdf/0910.3832.pdf

[17] M. Pireddu, F. Zanolin, Some remarks on fixed points for maps which are expansive along one direction, Rend. Istit. Mat. Univ. Trieste 39 (2007) 245274.

[18] M. Pireddu, F. Zanolin, Chaotic dynamics in the Volterra predator-prey model via linked twist maps, Opuscula Math. 28/4 (2008) 567-592.

[19] H.A. Simon, Reason in Human Affairs, Stanford University Press, Stanford, 1983.

[20] P. Walters, An Introduction to Ergodic Theory, Graduate Texts in Mathematics, Vol. 79, Springer-Verlag, New York, 1982.

[21] S. Wiggins, Introduction to Applied Nonlinear Dynamical Systems and Chaos, Texts in Applied Mathematics, Springer-Verlag, New York, 1990.

[22] U. Witt, The Evolving Economy: Essays on the Evolutionary Approach to Economics, Edward Elgar, Cheltenham, 2003.

[23] W. Wu, Z. Chen, W.H. Ip, Complex nonlinear dynamics and controlling chaos in a Cournot duopoly economic model, Nonlinear Analysis: Real World Applications 11 (2010) 4363-4377. 